\title{
Fast Convergence Cooperative Dynamic Spectrum Access for Cognitive Radio Networks
}

\author{
Tapiwa M. Chiwewe, Member, IEEE, and Gerhard P. Hancke, Senior Member, IEEE
}

\begin{abstract}
Cognitive radio and dynamic spectrum access can reform the way that radiofrequency spectrum is accessed. Problems of spectrum scarcity, coexistence and unreliable wireless communication that affect industrial wireless networks can be addressed. In this paper, a game theoretic dynamic spectrum access algorithm that improves upon on a hedonic coalition formation algorithm for spectrum sensing and access is presented. The modified algorithm is tailored for faster convergence and scalability and makes use of a novel simultaneous multi-channel sensing and access technique. Results to demonstrate the performance improvements of the adapted algorithm are presented and the use of different decision rules are investigated revealing that a conservative decision rule for exploiting spectrum opportunities performs better than an aggressive decision rule in most scenarios. The algorithm that was developed could be a key enabler for future cognitive radio networks.
\end{abstract}

Index Terms - cognitive radio, industrial wireless, spectrum management.

\section{INTRODUCTION}

$\mathrm{T}$ he radiofrequency spectrum is regulated by government agencies worldwide, using a fixed spectrum assignment policy, whereby usage of spectrum is controlled and certain sections of the spectrum are assigned to license holders or to certain services [1]. Most of the spectrum is used on an onand-off basis, and usage tends to be congested in certain sections of the spectrum. There are cases of real and artificial spectrum scarcity [2] that call for more efficient use of radiofrequency spectrum.

The situation, in which spectrum is used inefficiently, can be overcome by adopting opportunistic approaches to accessing spectrum. These approaches to reforming the way spectrum is accessed are referred to as Dynamic Spectrum Access (DSA) strategies. DSA is an important feature of Cognitive Radio (CR), a revolutionary new communication paradigm where wireless radios can sense, learn and adapt. Cognitive radio networks (CRNs) show promise in providing good quality service communication wherever and whenever required, be it in the home, the office or harsh industrial

Manuscript received July 2, 2017; accepted November 14, 2017.

T. M. Chiwewe, and G. P. Hancke are with the Department of Electrical, Electronic and Computer Engineering, University of Pretoria, Pretoria, South Africa (e-mail: tapiwa.chiwewe@ieee.org; ghancke@ieee.org)

T. M. Chiwewe is also with IBM Research, Johannesburg, South Africa.

G. P. Hancke is also with the Department of Computer Science, City University of Hong Kong, Hong Kong. networks [3]. CRs enable the co-existence of co-located dissimilar wireless networks that must operate reliably and with minimal latency, as is the case in industrial wireless networks and smart utility networks. These capabilities are key for applications such as factory and industrial automation, distributed control systems, automotive systems and process monitoring [3, 4]. CR is suitable for challenging environments such as underground monitoring [5], tele-intensive care units [6] or ambient environment monitoring [7].

CR allows unlicensed, secondary users (SUs) to use spectrum that has been allocated to licensed, primary users (PUs) opportunistically. This is particularly attractive in cases where PUs are not using the full spectrum, which results in spectrum opportunities known as spectrum holes or whitespaces. Different methods can be devised for CRs to make use of spectrum holes and share spectrum. Game theory lends itself to the problem of spectrum sharing in CRNs, as it provides an established structure to analyse the action and behavior of network users; this arises out of the fact that game theory is a mathematical tool for analyzing strategic interactions between several decision makers [8]. It also makes available measures that can be used to determine the most desirable solution to spectrum sharing.

Game theory has been used extensively in CR and in all categories of games: cooperative, non-cooperative, stochastic, economic, auction and mechanism design. Game theory was used for energy efficient communications in multi-hop networks in [9]. It has been used for spectrum pooling, using a joint optimisation framework [10]. In [11] and [12], cooperative spectrum sharing was investigated. The case of spectrum access using game theory was investigated using a pricing based model in [13] and surveyed in [14]. Sharing in MIMO CRNs using cooperative game theory was investigated in [15]. Power control is another application of game theory in $\mathrm{CR}$, as shown in [16] and [17]. Game theory has also been used for CR security: in [18] and [19], it was used for antijamming defense; in [20], it was used for intelligent jamming of CR. A Bayesian hierarchical mechanism design for CRNs was studied in [21] and a Stackelberg model for opportunistic sensing was developed by [22]. Joint sensing and access has also been studied, for example in: [23], where an evolutionary game was used; and in [24], where Bayesian social learning with negative externality was investigated.

In this paper, a scalable fast converging dynamic spectrum access algorithm is developed, which allows for opportunistic spectrum access. It builds and improves on a previously 
developed hedonic coalition formation game for cooperative spectrum sensing and channel access [25]. The original algorithm was attractive in that it is more practical than other previous work, considers multiple licensed channel scenarios as opposed to just single licensed channel scenarios, and takes energy consumption into account. In addition, it makes us of cooperative spectrum sensing which outperforms local spectrum sensing approaches. The contribution of this paper is:

- Direct comparison of two different decision rules, the conservative OR rule which prioritises protecting PUs, and the aggressive AND rule which maximises spectrum access opportunities for SUs. In cases where the number of licensed channels and SUs are closely matched it is possible for the OR rule to provide better average utility. We show that in cases where the number of SUs is considerably larger than the number of licensed channels then the OR rule results in higher average utility.

- A proposed fast convergence spectrum sensing and access algorithm that is scalable and can reach the Nash-stable partition of coalitions much faster than the original algorithm on which it is based. This is through a novel technique for contention free simultaneous multiplechannel sensing and access. Each node senses and accesses spectrum if it has the highest priority to do so in both it's incumbent channel and target channel. Fast convergence is important to achieve high scalability and has not been comprehensively addressed in past work.

\section{System Model}

\section{A. Network Architecture}

The network architecture is composed of two types of users: PUs that are licensed; and SUs that are unlicensed transmitterreceiver pairs. The SUs opportunistically use spectrum bands that are licensed for use by PUs. The spectrum bands are divided into a number of channels with each having a fixed frequency bandwidth. There are $C$ non-overlapping licensed channels in total and $D$ SUs in the network $(D \geq C)$. The set of PUs is $\mathcal{P} \in\{1, \ldots, C\}$ and the set of SUs is $\mathcal{D} \in\{1, \ldots, D\}$. Each licensed channel has a PU operating in it that can either be active or inactive - denoted by $P U_{i}, i \in \mathcal{P}$ - and the channel bandwidth is $B_{i}$. An example network configurations is shown in Fig. 1.

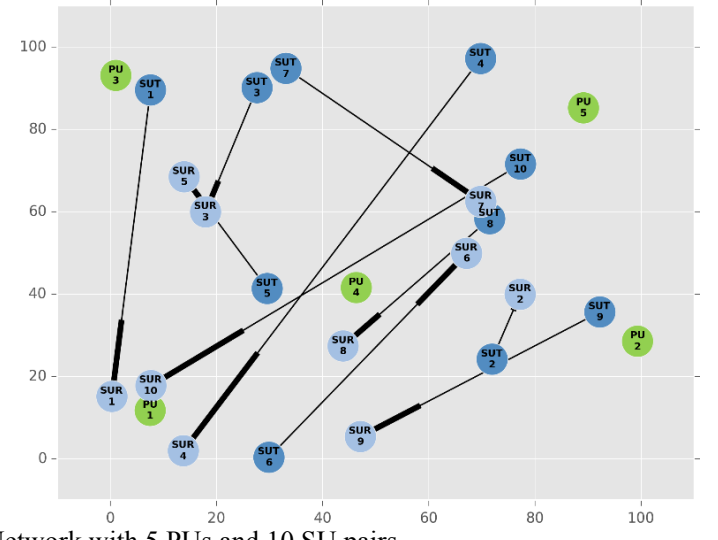

Fig. 1. Network with 5 PUs and 10 SU pairs.

\section{B. Channel Occupancy Model}

The status of each channel is represented using an ON-OFF usage channel model in which the channel switches status between ON (busy) and OFF (idle). To detect spectrum holes and avoid causing interference to PUs, it is necessary for SUs to perform spectrum sensing before transmission, a SU can only access a channel when the channel is in the OFF state. The transition rate for channel $i$ from the ON to the OFF state is denoted by $\eta_{i}$ and $\rho_{i}$ denotes the opposite transition. The sojourn times in the ON and OFF states for channel $i$ are $\bar{T}_{O N}^{i}=1 / \eta_{i}$ and $\bar{T}_{O F F}^{i}=1 / \rho_{i}$. The status of a given channel, $i$, is described in the Markov chain shown in Fig. 2.

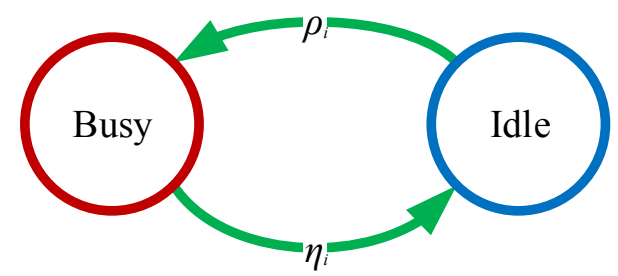

Fig. 2. Markov chain for channel status.

The SUs are located in the vicinity of the PUs and each SU is denoted by $S U_{j}, j \in \mathcal{D}$. A SU accesses one of the $C$ channels only when it is idle. If $P U_{i}$ is present in the channel of interest $i, i \in \mathcal{P}$ this is denoted by $H_{1, i}$; if $P U_{i}$ is absent, this is denoted by $H_{0, i}$. An assumption is made that a $\mathrm{SU}$ always has data to send. Another assumption is that each user has a single transceiver that operates in half duplex mode in a single channel at any instance. Each SU is required to have spectrum agility and to be capable of dynamic frequency selection and able to change channels with minimal latency. The probability that $P U_{i}$ is active is denoted as $P_{H_{1, i}}$; the probability that it is idle is denoted as $P_{H_{0, i}}$. It follows that $P_{H_{1, i}}+P_{H_{0, i}}=1$.

\section{SPECTRUM SENSING}

\section{A. Individual Spectrum Sensing}

To determine the status of channels, spectrum sensing is performed. Energy detection is used as the spectrum sensing technique, due to its low implementation complexity and minimal runtime overhead [26]. When using energy detection for spectrum sensing, and given a detection threshold $\varepsilon$ with test statistic $y$, the probability of detection and the probability of false alarm are defined as:

$$
\begin{aligned}
& P_{d}=\operatorname{Pr}\left(y>\varepsilon \mid H_{1, i}\right), \\
& P_{f}=\operatorname{Pr}\left(y>\varepsilon \mid H_{0, i}\right) .
\end{aligned}
$$

The case under consideration is that of complex valued Phase Shift keying (PSK) signals, Circularly Symmetric Complex Gaussian (CSCG) noise that is an independent and identically distributed random process in each channel $i, i \in \mathcal{P}$ with a mean of zero and a variance of $\sigma_{n, i}^{2}$ [27]. The bandwidth of each channel is $B_{i}$ and the power spectral density is $N_{0}$, which gives $\sigma_{n, i}^{2}=N_{0} B_{i}$. Each PU signal $P U_{i}, i \in \mathcal{P}$ is an 
independent and identically distributed random process, with a zero mean and a variance of $\sigma_{s, i}^{2}$. The signal to noise ratio of $P U_{i}$ measured by a given secondary user, $S U_{j}, j \in \mathcal{D}$, under the hypothesis $H_{1, i}$; an average channel gain between $P U_{i}$ and $S U_{j}$ of $\left|g_{i, j}\right|^{2}$ is given by $\gamma_{i, j}=\left|g_{i, j}\right|^{2} \sigma_{s, i}^{2} / \sigma_{n, i}^{2}$.

Narrowband sensing is considered where each SU senses one channel per time frame. Each frame has a duration of $T$ and each SU has a sensing duration $\delta$, which is non-zero and less than $T$. The time left for data transmission is therefore $T-$ $\delta$. The sampling frequency for received signals is $f_{s}$ and the sensing duration and frame duration are multiples of the sample time, i.e. $\frac{1}{f_{s}}$. It follows that the number of samples during spectrum sensing is $\delta f_{s}$.

Given the above, the probability of false alarm for a channel $i, i \in \mathcal{P}$ and $S U_{j}, j \in \mathcal{D}$ is given by

$$
\begin{aligned}
P_{f, i, j}\left(\varepsilon, \delta, \sigma_{n, i}^{2}\right) & =\operatorname{Pr}\left(y_{j, i}>\varepsilon \mid H_{0, i}\right) \\
& =Q\left(\left(\varepsilon / \sigma_{n, i}^{2}-1\right) \sqrt{\delta f_{s}}\right) .
\end{aligned}
$$

In the above equation: $Q($.$) is the complementary$ distribution function of the standard Gaussian; and $y_{j, i}$ is the test statistic for the energy detector of $S U_{j}$ in channel $i \in \mathcal{P}$. Given the same sensing duration $\delta$ and detection threshold $\varepsilon$ for all SUs and the same bandwidth $B_{i}$ for all PUs, then the probability of false alarm $P_{f, i, j}\left(\varepsilon, \delta, \sigma_{n, i}^{2}\right)$ is the same for all channels and all SUs, i.e. $\forall i \in \mathcal{P}, \forall j \in \mathcal{D}$. The probability of detection for a channel $i, i \in \mathcal{P}$ and $S U_{j}, j \in \mathcal{D}$ given by

$$
\begin{aligned}
& P_{d, i, j}\left(\varepsilon, \delta, \sigma_{n, i}^{2}, \gamma_{i, j}\right)=\operatorname{Pr}\left(y_{j, i}>\varepsilon \mid H_{1, i}\right) \\
= & Q\left(\left(\varepsilon / \sigma_{n, i}^{2}-\gamma_{i, j}-1\right) \sqrt{\left.\delta f_{s} /\left(2 \gamma_{i, j}+1\right)\right)} .\right.
\end{aligned}
$$

The receiver operating characteristic (ROC) curve for energy detector based spectrum sensing is shown in: Fig. 3 for different SNR values; Fig. 4 for different values of sensing duration, where a higher sensing duration implies that a higher number of samples is collected. It can be seen that a higher SNR and a greater number of samples results in better performance of the energy detector.

\section{B. Cooperative Spectrum Sensing}

Cooperative Spectrum Sensing (CSS), where SUs cooperate with one another, can be used to increase the performance of spectrum sensing [28]. CSS can help overcome the problems of fading, shadowing and hidden terminals. Each channel has a decision node (DN) that is selected in each time frame on expiry of the sensing period. The decision node is selected from among the SUs in a given channel $i$, the set of which is denoted by $\mathcal{A}_{i}$ with $\mathcal{A}_{i} \subseteq \mathcal{D}, \forall i \in \mathcal{P}$ and $\bigcup_{i \in \mathcal{P}} \mathcal{A}_{i}=\mathcal{D}$. $D N_{i}, i \in \mathcal{P}$ is responsible for determining whether the channel $i$, is busy or idle, based on sensing results from other SUs that sense the channel. Different decision fusion rules can be used including the: AND rule, OR rule, soft combination rule, majority rule, and the $k$-out-of- $n$ rule [29]. The $k$-out-of- $n$ rule becomes the AND rule, OR rule and majority rule when $k$ is $1, n$ and $\geq n / 2$ respectively.

Considering the OR rule, if at least one SU presumes that a
$\mathrm{PU}$ is present in a channel, then the channel is considered to be busy. In this case, for channel $i, i \in \mathcal{P}$, the probability of detection and the probability of false alarm are defined as

$$
\begin{gathered}
P_{f, i}=1-\prod_{j \in \mathcal{A}_{i}}\left(1-P_{f, i, j}\left(\varepsilon, \delta, \sigma_{n, i}^{2}\right)\right), \\
P_{d, i}=1-\prod_{j \in \mathcal{A}_{i}}\left(1-P_{d, i, j}\left(\varepsilon, \delta, \sigma_{n, i}^{2}, \gamma_{i, j}\right)\right) .
\end{gathered}
$$

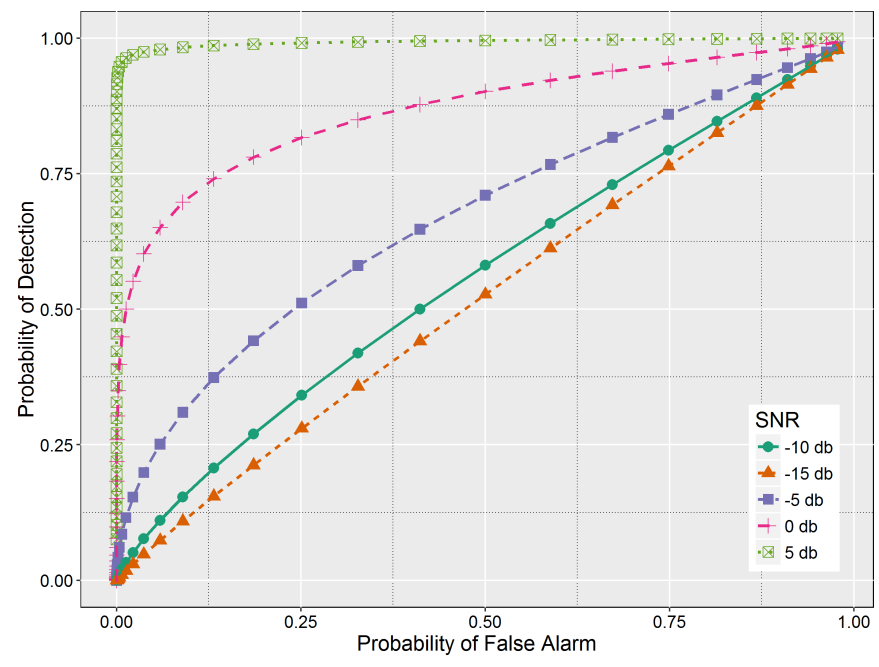

Fig. 3. ROC for spectrum sensing at different values of SNR.

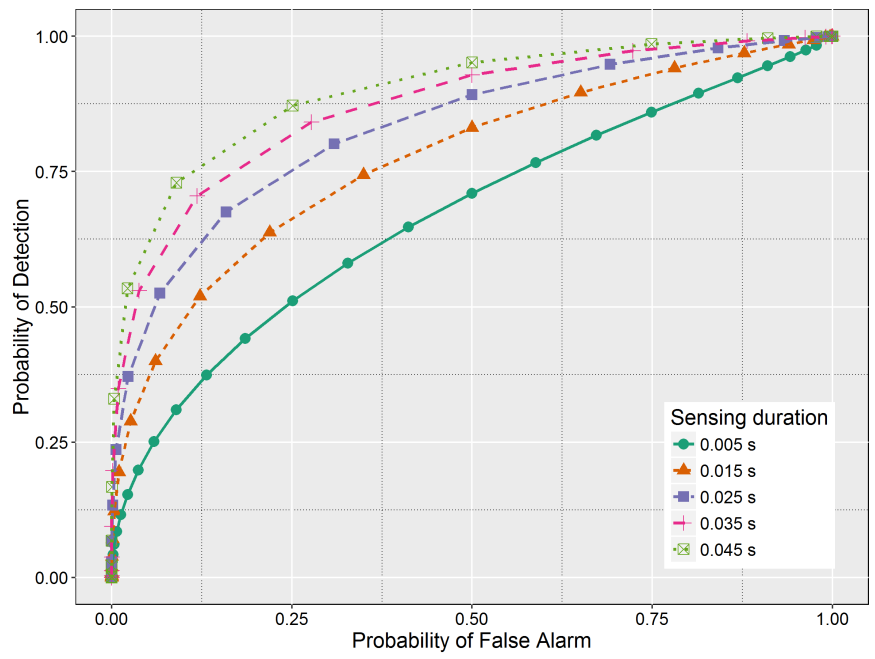

Fig. 4. ROC for spectrum sensing at different values of sensing duration.

Considering the AND rule, there needs to be consensus among SUs that a PU is present in a channel to consider it as being busy. For a channel $i, i \in \mathcal{P}$ the probability of detection and probability of false alarm are defined as

$$
\begin{gathered}
P_{f, i}=\prod_{j \in \mathcal{A}_{i}}\left(P_{f, i, j}\left(\varepsilon, \delta, \sigma_{n, i}^{2}\right)\right), \\
P_{d, i}=\prod_{j \in \mathcal{A}_{i}}\left(P_{d, i, j}\left(\varepsilon, \delta, \sigma_{n, i}^{2}, \gamma_{i, j}\right)\right) .
\end{gathered}
$$

The OR rule is suitable for a conservative approach where high priority is placed on protecting PUs, whereas the AND 
rule is suitable for an aggressive approach that seeks to maximise spectrum access opportunities for SUs. Making use of the OR rule gives a higher probability of detection, whereas making use of the AND rule gives a lower probability of false alarm. If $D N_{i}$ decides that $P U_{i}$ is active, then all SUs, $S U_{j}, \forall j \in \mathcal{A}_{i}$ operating in the channel are not permitted to transmit during the time-frame period. If, on the other hand, $D N_{i}$ decides that the channel is idle, then all the SUs operating in the channel have an equal opportunity to access the channel, i.e. the probability of transmitting for each $\mathrm{SU}, S U_{j}, \forall j \in \mathcal{A}_{i}$ is $1 /\left|\mathcal{A}_{i}\right|$.

\section{SPECTRUM SHARING}

\section{A. Hedonic Coalition Formation}

In [25], the authors present a multi-channel coalition formation game for spectrum sensing and access, which terminates at a final partition that is individually stable and Nash stable. Considering a channel $i$ with a SU transmit power of $P_{s u}$, a SU sensing power of $P_{s s}$, a noise power of $\sigma_{n, i}^{2}$, and an average channel gain of $\left|h_{j, i}\right|$ between a SU transmitter and receiver pair $j$ in channel $i$, the rate of transmission can be represented as [25]:

$$
R_{j, i}=B \log _{2}\left(1+\left|h_{j, i}\right|^{2} \frac{P_{s u}}{\sigma_{n, i}^{2}}\right) \text {. }
$$

For a set $\mathcal{A}_{i}$, the payoff is the difference between the reward and the penalty, where: the reward is defined as the amount of data that is transmitted by the SUs in $\mathcal{A}_{i}$; and the penalty is given by $\lambda$, where $\lambda>0$ is defined as the unity penalty factor per second that is applied when a PU transmission is interfered with. The 4 possible scenarios in channel $i$ regarding the decision of $D N_{i}$ and the activity of $P U_{i}$ are:

1. $D N_{i}$ makes a decision that channel $i$ is idle and $P U_{i}$ is silent, i.e. a true negative, which occurs with probability $P_{0 \mid 0, i}=P_{H_{0, i}}\left(1-P_{f, i}\right)$.

2. $D N_{i}$ makes a decision that channel $i$ is busy and $P U_{i}$ is silent, i.e. a false positive, which occurs with probability $P_{1 \mid 0, i}=P_{H_{0, i}} P_{f, i}$.

3. $D N_{i}$ makes a decision that channel $i$ is idle and $P U_{i}$ is active, i.e. a false negative, which occurs with probability $P_{0 \mid 1, i}=P_{H_{1, i}}\left(1-P_{d, i}\right)$.

4. $D N_{i}$ makes a decision that channel $i$ is busy and $P U_{i}$ is active, i.e. a true positive, which occurs with probability $P_{1 \mid 1, i}=P_{H_{1, i}} P_{d, i}$.

In the first scenario, which corresponds to a true negative decision by the decision node, the SU transmission proceeds successfully and there is no penalty, since no PU transmission is interfered with. The payoff in this scenario for set $\mathcal{A}_{i}$ is $r_{0 \mid 0}\left(\mathcal{A}_{i}\right)=\frac{\left(\sum_{j \in \mathcal{A}_{i}} R_{j, i}\right)(T-\delta)}{\mid \mathcal{A}_{i \mid}}$ and the energy consumption for the set is $e_{0 \mid 0}\left(\mathcal{A}_{i}\right)=P_{s s}\left|\mathcal{A}_{i}\right| \delta+P_{s u}(T-\delta)$.

In the second scenario, which corresponds to a false positive decision being made by the decision node, there is no SU transmission, resulting in no reward, and there is no penalty, since no $\mathrm{PU}$ transmission is interfered with. The payoff in this scenario for set $\mathcal{A}_{i}$ is $r_{1 \mid 0}\left(\mathcal{A}_{i}\right)=0$ and the energy consumption for the set is $e_{1 \mid 0}\left(\mathcal{A}_{i}\right)=P_{S S}\left|\mathcal{A}_{i}\right| \delta$.

In the third scenario, which corresponds to a false negative decision being made by the decision node, the SU transmission proceeds at the same time as PU transmission and they interfere with one another. As a result, there is no reward and a penalty is applied. The payoff in this scenario for set $\mathcal{A}_{i}$ is $r_{0 \mid 1}\left(\mathcal{A}_{i}\right)=-\lambda(T-\delta)$ and the energy consumption for the set is $e_{0 \mid 1}\left(\mathcal{A}_{i}\right)=P_{s s}\left|\mathcal{A}_{i}\right| \delta+P_{s u}(T-\delta)$.

In the fourth scenario, which corresponds to a true positive decision being made by the decision node, there is no SU transmission and PU transmission proceeds with no interference. As a result, there is no reward or penalty. The payoff in this scenario for set $S_{i}$ is $r_{1 \mid 1}\left(\mathcal{A}_{i}\right)=0$ and the energy consumption for the set is $e_{1 \mid 1}\left(\mathcal{A}_{i}\right)=P_{s s}\left|\mathcal{A}_{i}\right| \delta$.

The payoff that is expected in each time-frame, when considering all four scenarios, is:

$$
\begin{array}{r}
r\left(\mathcal{A}_{i}\right)=\sum_{a=0}^{1} \sum_{b=0}^{1} P_{a \mid b, i} r_{a \mid b}\left(\mathcal{A}_{i}\right) \\
=P_{0 \mid 0, i} \frac{\sum_{j \in \mathcal{A}_{i}} R_{j, i}}{\left|\mathcal{A}_{i}\right|}(T-\delta) \\
\quad-P_{0 \mid 1, i} \lambda(T-\delta) .
\end{array}
$$

The expected energy consumption per time-frame is as follows, when taking into account all four scenarios:

$$
\begin{aligned}
e\left(\mathcal{A}_{i}\right) & =\sum_{a=0}^{1} \sum_{b=0}^{1} P_{a \mid b, i} e_{a \mid b}\left(\mathcal{A}_{i}\right) \\
& =P_{S S}\left|\mathcal{A}_{i}\right| \delta+\left(P_{0 \mid 0, i}+P_{0 \mid 1, i}\right) P_{s u}(T-\delta) .
\end{aligned}
$$

The value function of set $\mathcal{A}_{i}$ is defined as the ratio of the expected payoff to the expected energy consumption; this is given by:

$$
\begin{aligned}
& v\left(\mathcal{A}_{i}\right) \triangleq \frac{r\left(\mathcal{A}_{i}\right)}{e\left(\mathcal{A}_{i}\right)} \\
& =\frac{P_{0 \mid 0, i} \sum_{j \in \mathcal{A}_{i}} R_{j, i}(T-\delta)-\left|\mathcal{A}_{i}\right| P_{0 \mid 1, i} \lambda(T-\delta)}{\left|\mathcal{A}_{i}\right|\left(P_{s s}\left|\mathcal{A}_{i}\right| \delta+\left(P_{0 \mid 0, i}+P_{0 \mid 1, i}\right) P_{s u}(T-\delta)\right)} .
\end{aligned}
$$

Since all SUs in a given channel sense and access the channel with the same probability, the utility that they receive is the same, and the utility function is defined as:

$$
u_{j}^{\mathcal{A}_{i}}=\frac{v\left(\mathcal{A}_{i}\right)}{\left|\mathcal{A}_{i}\right|} .
$$




\section{B. Coalition Formation Algorithm}

The problem of cooperative multi-channel spectrum sensing and access can be modeled using coalition game theory. The set $\mathcal{A}_{i}$ represents coalition $i$ and there are $C$ coalitions in total. Each SU is a member of one coalition and there is one coalition per channel. The basic components of the game are as follows:

- A set of $\mathcal{D}$ players, which are the $\operatorname{SUs} S U_{j}, j \in \mathcal{D}$.

- A set of strategies for each $\mathrm{SU}$, which is the channel it decides to sense and access.

- A utility function, which is given in (12) for each SU.

The algorithm for channel sensing and access is given below.

\begin{tabular}{l}
\hline Algorithm 1 Coalition formation algorithm \\
Executed by $S U_{j}, j \in \mathcal{D}$ \\
\hline $1: \quad$ Initialisation: $\mathcal{A}_{1}^{0}:=\mathcal{D}, \mathcal{A}_{l}^{0}:=\emptyset, \forall l \in \mathcal{P} \backslash\{1\}$ \\
$2: \quad$ for iteration $\mathrm{r}:=1$ to $L I M I T$ do \\
$3: \quad \mathcal{A}_{i}^{(r)}:=\mathcal{A}_{i}^{(r-1)}, \beta_{j}:=i$, where $i \in \mathcal{P}$ and $j \in \mathcal{A}_{i}^{(r)}$ \\
4: $\quad S U_{j}$ broadcasts its measured SNR information $\gamma_{i, j}^{(r)}$ and \\
$\quad$ transmission rate $R_{j, i}$ to other SUs, $S U_{n}, \forall n \in \mathcal{A}_{i}^{(r)} \backslash\{j\}$
\end{tabular}

5: $S U_{j}$ receives the measured SNR information $\gamma_{i, n}^{(r)}$ and transmission rate $R_{n, i}$ from other $S U_{n}, \forall n \in \mathcal{A}_{i}^{(r)} \backslash\{j\}$

6: $\quad \mathrm{s}:=\arg \max _{t \in \mathcal{A}_{i}^{(r)}} \gamma_{i, t}^{(r)}$

7: $\quad D N_{i}^{(r)}:=S U_{S}$

8: $S U_{j}$ generates $\theta_{j}$ which is a standard normal random variable

9: $\quad S U_{j}$ selects a random channel $\alpha_{j}$ other than its current channel $\beta_{j}$ such that $\alpha_{j} \in \mathcal{P}$ and $\alpha_{j} \neq \beta_{j}$

10: $S U_{j}$ broadcast details on $\theta_{j}, \alpha_{j}$ and $\beta_{j}$ to other SUs, $S U_{k}, \forall k \in \mathcal{D}, k \neq j$

11: $S U_{j}$ receives details on $\theta_{k}, \alpha_{k}$ and $\beta_{k}$ from other SUs, $S U_{k}, \forall k \in \mathcal{D}, k \neq j$

12: $\quad S U_{j}$ determines $\mathcal{A}_{l}^{(r)} \forall l \in \mathcal{P}, l \neq i$ from $\beta_{k}, \forall k \in$ $\mathcal{D}, k \neq j$

13: $\mathrm{m}:=\arg \max _{w} \theta_{w}, \forall w \in \mathcal{A}_{i}^{(r)}$

14: $\quad$ if $S U_{j}=S U_{m}$ then

15: $\quad z:=\varnothing$

16: $\quad Z:=Z \cup\{q\}, \quad \mathrm{q}:=\arg \max _{\mathrm{q}} \theta_{q}, \forall q \in \mathcal{A}_{l}^{(r)}, \forall l \in$ $\mathcal{P}, l \neq i$

17: $\quad \mathcal{Z}:=\mathcal{Z} \backslash\{p\}, \forall p \in \mathcal{Z}, \theta_{p}<\theta_{j}$

18: $\quad$ if $\beta_{j} \neq \alpha_{k} \forall k \in Z$ then

19: $\quad$ if $\alpha_{j} \neq \alpha_{k} \forall k \in Z$ then

20: $\quad S U_{j}$ computes $u_{j}^{\mathcal{A}_{i}^{(r)}}:=v\left(\mathcal{A}_{i}^{(r)}\right) /\left|\mathcal{A}_{i}^{(r)}\right|$

21: $\quad \mathcal{A}_{i}^{(r)}:=\mathcal{A}_{i}^{(r)} \backslash\{j\}$

22: $\quad S U_{j}$ requests and obtains the information of
23:

24:

25:

26:

27:

28 :

30 :

31:

32:

33:

34:

35:

36:

37:

38:

$$
\mathcal{A}_{\alpha_{j}}^{(r)} \text { from } D N_{\alpha_{j}}^{(r)}
$$

$$
\mathcal{A}_{\alpha_{j}}^{(r)}:=\mathcal{A}_{\alpha_{j}}^{(r)} \cup\{j\}
$$$$
\text { if } \mathcal{A}_{\alpha_{j}}^{(r)} \in h(j) \text { then }
$$$$
\mathcal{A}_{\alpha_{j}}^{(r)}:=\mathcal{A}_{\alpha_{j}}^{(r)} \backslash\{j\}
$$$$
\mathcal{A}_{i}^{(r)}:=\mathcal{A}_{i}^{(r)} \cup\{j\}
$$

else

$$
S U_{j} \text { computes } u_{j}{ }^{\left(\mathcal{A}_{j}\right.}\left(=v\left(\mathcal{A}_{\alpha_{j}}^{(r)}\right) /\left|\mathcal{A}_{\alpha_{j}}^{(r)}\right|\right.
$$

$$
\text { if } u_{j}^{\mathcal{A}_{\alpha_{j}}^{(r)}} \leq u_{j}^{\mathcal{A}_{i}^{(r)}} \text { then }
$$

$$
\mathcal{A}_{\alpha_{j}}^{(r)}:=\mathcal{A}_{\alpha_{j}}^{(r)} \backslash\{j\}
$$

end if

$$
\mathcal{A}_{i}^{(r)}:=\mathcal{A}_{i}^{(r)} \cup\{j\}
$$

\section{end if}

end if

\section{end if}

\section{end if}

$S U_{j}$ adds its current coalition to $h(j)$

end for

The algorithm above provides some improvements to the hedonic coalition formation algorithm on which it is based. The key improvement is faster convergence time. This is achieved by allowing multiple SUs to perform channel sensing and access during one time frame. Priority is based on the generation of a standard normal random variable, with a higher number denoting higher priority. The original algorithm allows only the SU with the highest priority to sense and access a channel in a time frame. The updated algorithm allows any SU to do so provided:

1. It has the highest priority in its channel's coalition.

2. There is no SU with a higher priority that has selected its current channel as a target for sensing and access.

3. The target channel it has selected for sensing and access does not have a higher priority SU in its coalition.

Thus, multiple SUs can perform channel sensing and access in the same time frame resulting in greater spectrum usage and there is no contention or instability in the network. The updated algorithm involves more processing steps that the original one. A study on the computational complexity of the new and updated algorithms and other similar algorithms will be useful. During message passing, apart from SUs sharing a standard random standard normal number that they each generate, they also share details on their incumbent and target channels. The random number can be sent in single precision floating point format that requires 4 bytes and the channel information can be sent as 1 byte unsigned integers to support up to 256 channels. Therefore, an extra 2 bytes of information will be sent. For each time frame, there is an incumbent channel and a target channel for a SU that is part of a coalition, an example is shown in Fig. 5. for the original algorithm. 


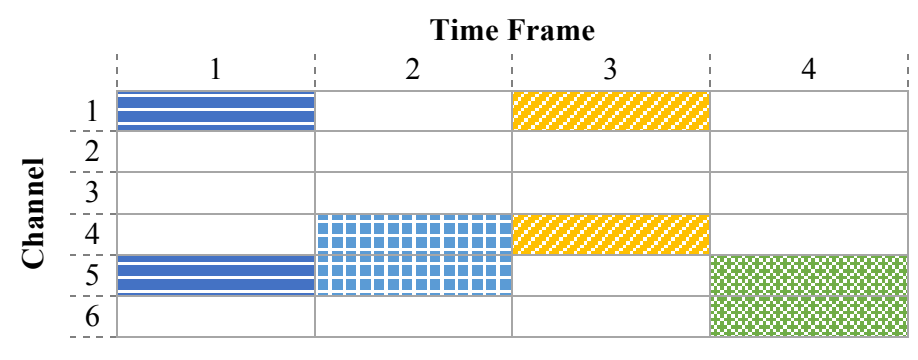

TABLE I

SIMULATION PARAMETERS

\begin{tabular}{lll}
\hline \hline Parameter & Description & Value \\
\hline$C$ & Number of channels & 50 \\
\hline$D$ & Number of secondary users & 100 \\
\hline$B$ & Channel bandwidth & $10 \mathrm{MHz}$ \\
\hline$T$ & Frame duration & $100 \mathrm{~ms}$ \\
\hline$f_{s}$ & Sample frequency & $1 \mathrm{kHz}$ \\
\hline$P_{s u}$ & SU transmit power & $100 \mathrm{~mW}$ \\
\hline$\sigma_{s, i}^{2}$ & PU transmit power & $100 \mathrm{~mW}$ \\
\hline$P_{s s}$ & SU sensing power & $100 \mathrm{~mW}$ \\
\hline$\varepsilon$ & Detector threshold & $0.2 \mathrm{~mW}$ \\
\hline$\sigma_{n, i}^{2}$ & Noise power & $0.1 \mathrm{~mW}$ \\
\hline$P_{H_{1, i}}$ & Probability $P U_{i}$ is active & 0.8 \\
\hline$\delta$ & Sensing duration & $5 \mathrm{~ms}$ \\
\hline$\lambda$ & Unit penalty per second & 100 \\
\hline$\left|g_{i, j}\right|^{2}$ & $\begin{array}{l}\text { Average channel gain between } P U_{i} \text { and } S U_{j} \\
\text { with a distance of } d_{i, j} \text { between them and a } \\
\text { path loss exponent } \gamma \text { set to } 2\end{array}$ & $\frac{1}{d_{i, j}^{\gamma}}$ \\
\hline$\left.h_{i, j}\right|^{2}$ & $\begin{array}{l}\text { Average channel gain between secondary } \\
\text { user transmitter receiver pair } S U_{j} \text { in channel } \\
i \text { with a distance of } d_{j, i} \text { between them and a } \\
\text { path loss exponent } \gamma \text { set to } 2\end{array}$ & $\frac{1}{d_{j, i}^{\gamma}}$ \\
\hline
\end{tabular}

Coalitions

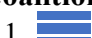

2

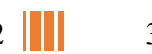

3 A

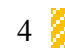

5

6

Fig. 5. Channel sensing and access by time frame with original algorithm.

An example for channel sensing and access when using the updated algorithm is shown in Fig. 6.

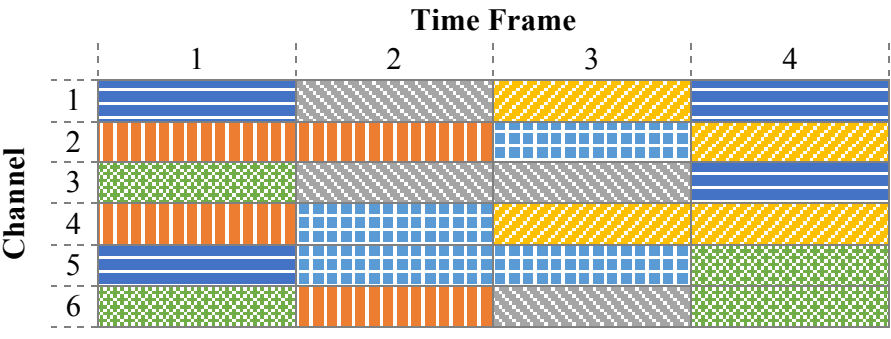

Coalitions

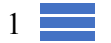

2

2

3
4820

5

\section{B. Coalitions}

The number of coalitions available to join corresponds with the number of channels available. Nodes in the same coalition operate in the same channel. Fig. 7 shows the coalitions that were formed for a network with 10 PUs and 30 SUs using the DSA-FC algorithm. The color of the node depicts the coalition the node is a part of.

Initially, all SUs are in the same coalition. As more iterations of the algorithm are run, the SUs get spread out among the different coalitions. The distribution of SUs among the different coalitions is shown in Fig. 8 for a network with 10 coalitions and $300 \mathrm{SUs}$ for the different algorithms. This is an indication of how many SUs are in each coalition, but does not show which SUs are in each coalition. The organisation of SUs across the coalitions is what the spectrum sensing and access algorithm seeks to optimise and is reflected in the average utility of the network. results were averaged. When investigating the effects of varying some of the simulation parameters, the algorithms were run to 200 iterations, where it was observed that they were not yet close to a point of equilibrium and the benefit of using one algorithm over the other was more pronounced.

A comparison follows below of the new fast convergence dynamic spectrum sensing and access algorithm (DSA-FC) to the original dynamic spectrum sensing and access (DSSA) algorithm [25]. A variation of the new algorithm that makes use of the AND decision rule (DSA-FC*) is also compared to the original algorithm with the same change in decision rule (DSSA*) 


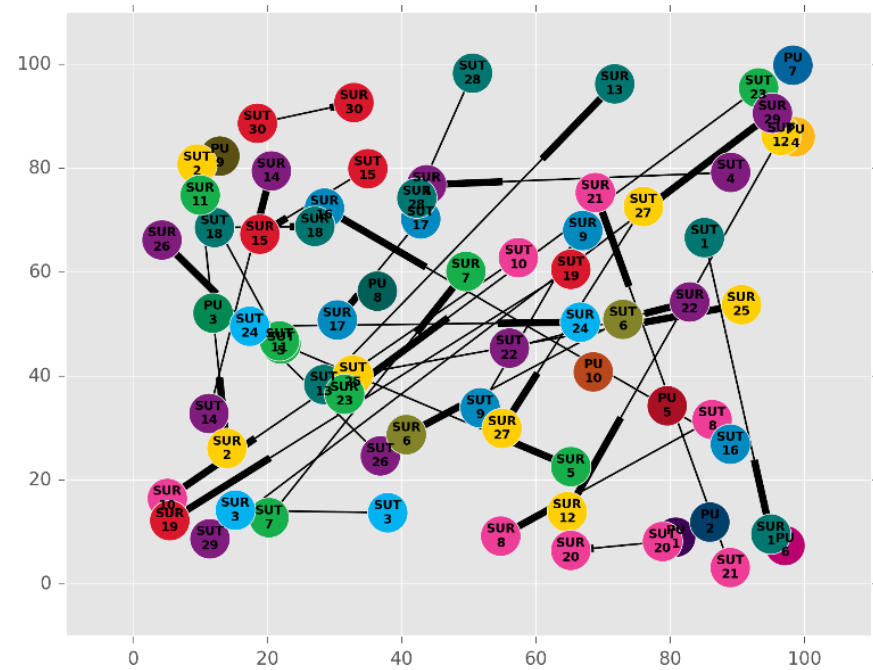

Fig. 7. Coalitions formed in network with 10 PUs and 30 SU pairs.

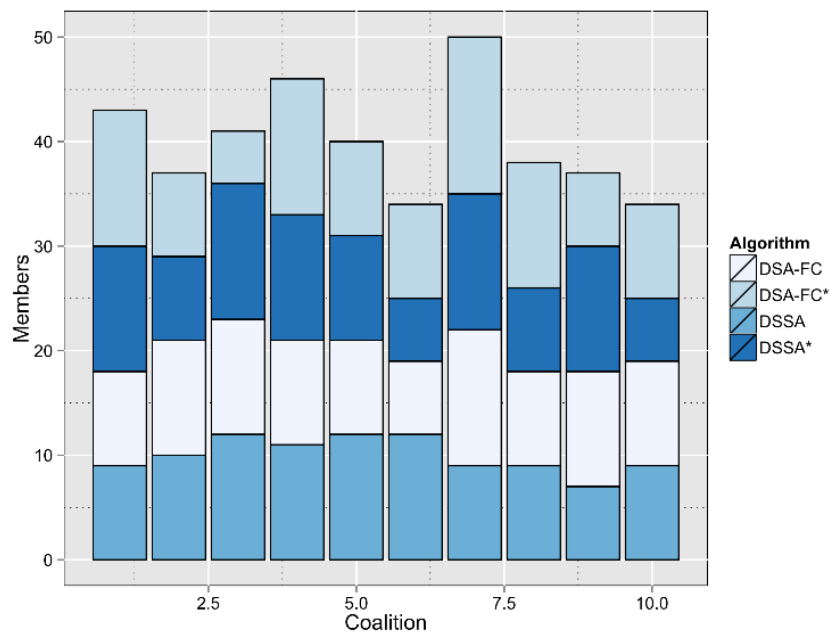

Fig. 8. Membership of coalitions formed in network with 10 PUs and $300 \mathrm{SU}$ pairs.

When considering how coalitions are formed, it can be seen that there is not an even distribution of SUs among the different coalitions; however, the number of SUs in each coalition is quite similar. This is likely because the utility that SUs will obtain is based on channel characteristics as well as coalition membership. A coalition in which the PU is far away from a particular SU is generally undesirable, because of the relatively low channel gain; however, should the channel be largely unoccupied, then this makes it attractive. There is, therefore, interplay between the channel gain and how congested a channel is. The net effect observed is that the Nash stable partition spreads SUs across the different channels fairly evenly, which suggests that SUs will be part of a coalition where the PU is closer to them and they receive a higher channel gain and therefore higher utility.

\section{Average Utility per Iteration}

The average SU utility per iteration is shown in Fig. 9. It can be seen that the adapted algorithm does converge faster than the original one. After several iterations, the partitions for both the adapted and the original algorithms are Nash stable and the utility is the same. The advantage here is fast convergence. When looking at the algorithm, this result it is to be expected, as going from just one SU performing channel sensing and access per time-frame to multiple SUs performing this action in a time-frame suggests that the coalition game should converge faster. Apart from fast convergence, the fact that the multi-user channel sensing and access is done in a way that ensures that there is no contention and instability in the coalition game is advantageous. What happens is that if a channel has members that are part of its coalition, one of them will have an opportunity to do sensing in that channel. This is in contrast with sensing being performed in one channel across the whole network. The adapted algorithm also ensures that there is no likelihood of a SU migrating to a channel that another SU has chosen to migrate to, which would result in contention and inaccurate expected utility in the new channel.

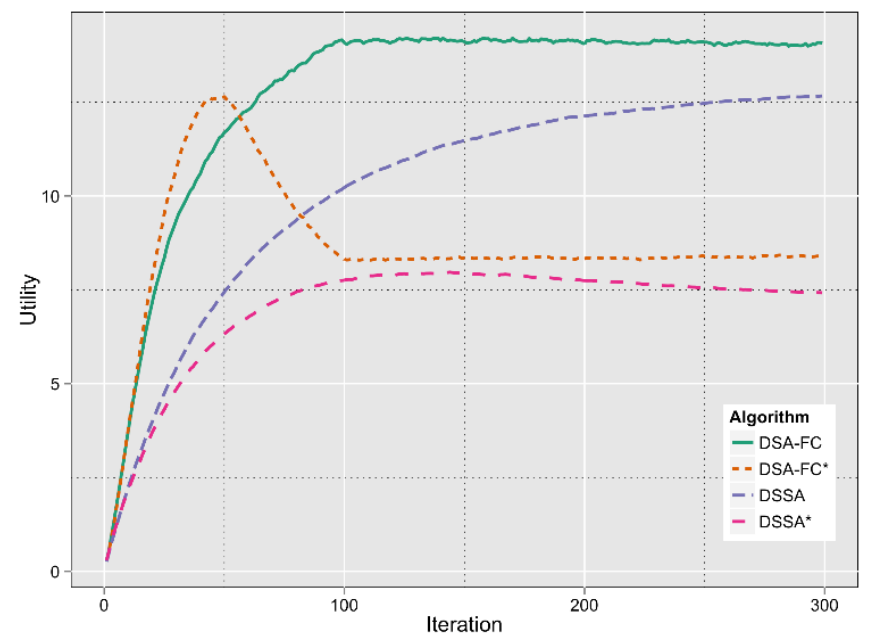

Fig. 9. Average SU utility vs. iteration.

The choice of decision rule is shown to have a marked difference in the results obtained. The OR rule is a more conservative rule to use that seeks to protect PUs more and it results in a much higher average utility than when using the AND rule. When using the AND rule, it can be seen that the fast convergence algorithm gives a rapid increase in utility in the early stages of the iterations, before dropping and finally settling to the Nash-stable partition. The highest utility is obtained before equilibrium is reached.

\section{Average Utility with Number of Channels}

The average SU utility for different number of channels is shown Fig. 10. The adapted fast convergence algorithm scales much better than the original one. Here the benefits of fast convergence are amplified. The original algorithm takes longer and longer to converge as the number of channels increases, and the difference in average utility after a given number of iterations keeps increasing as the number of channels increases. This shows clearly that the adapted algorithm scales well and out-performs the original one significantly in this regard. 


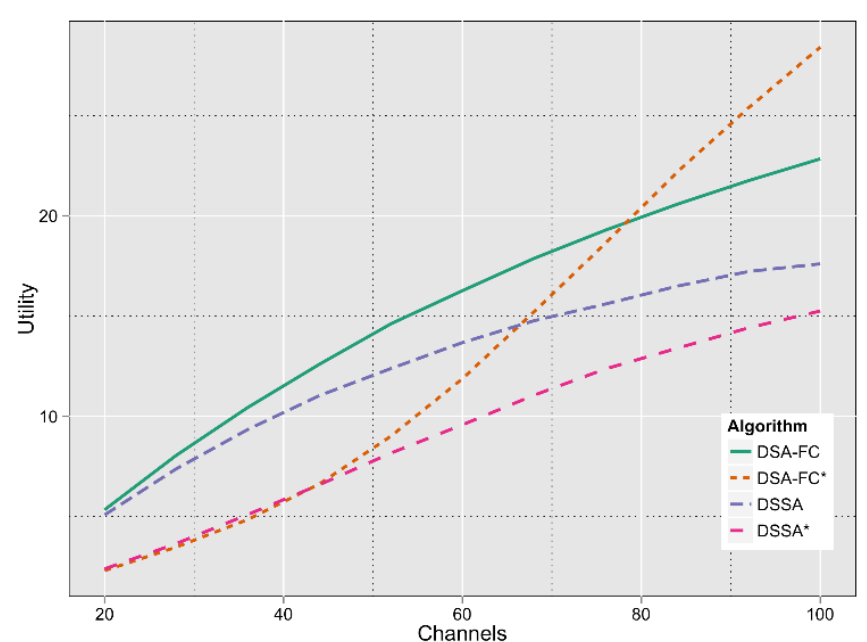

Fig. 10. Average SU utility vs. number of channels.

\section{E. Average Utility with Number of Secondary Users}

The average SU utility for different number of secondary users is shown in Fig. 11. When the number of SUs is low the adapted algorithm performs better but as the number of SUs increases the difference becomes less and eventually they converge to the same utility.

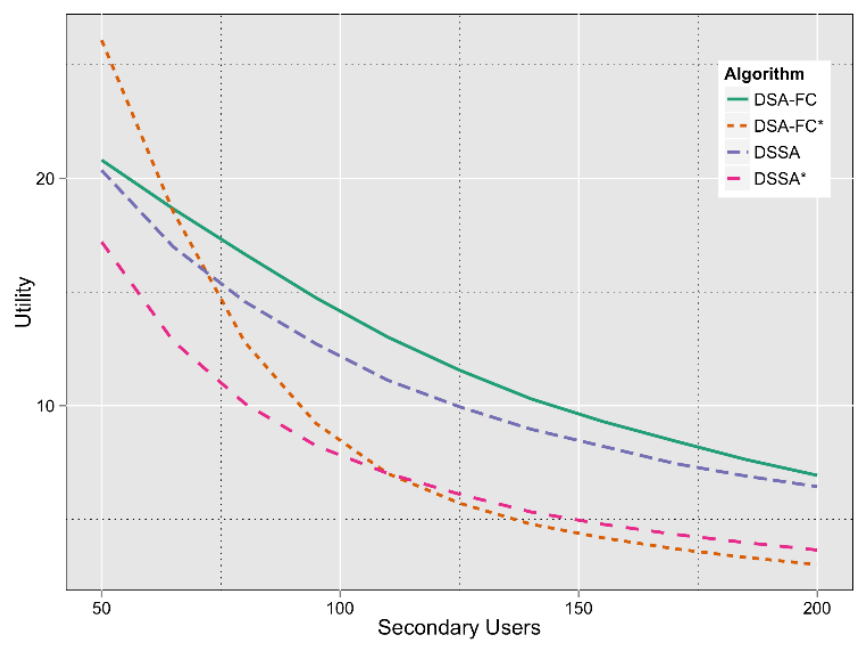

Fig. 11. Average SU utility vs. number of SU pairs.

\section{F. Average Utility with Probability of Primary User Activity}

The average SU utility for different probabilities of primary users being active is shown in Fig. 12. When the primary user activity is low, a higher average utility is achieved using the original algorithm, whereas when its high, the adapted algorithm performs better.

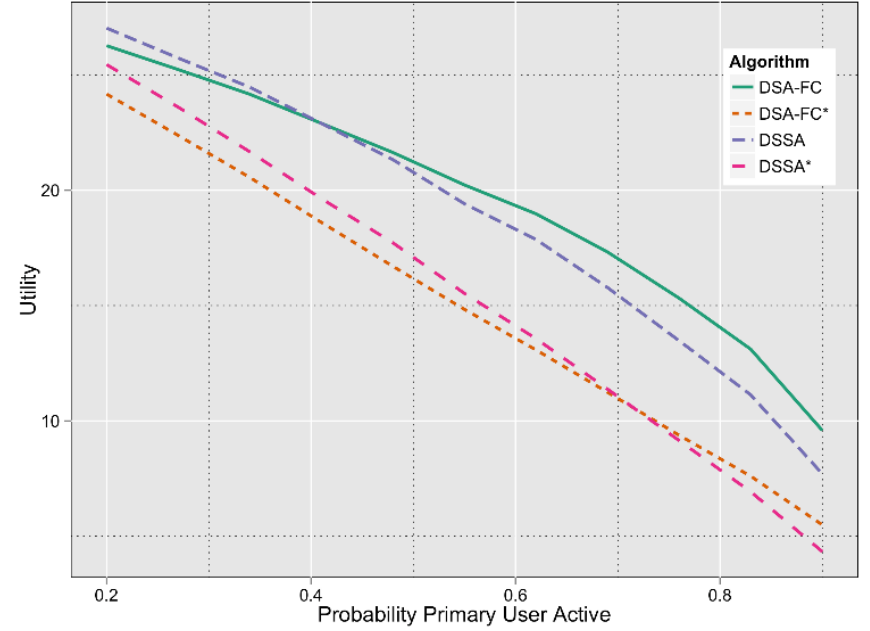

Fig. 12. Average SU utility vs. probability of primary users being active.

\section{G. Average Utility with Sensing Time}

The average SU utility for different sensing durations and thus different number of sensing samples collected is shown in Fig. 13. The sensing duration seems to have a minimal effect when comparing the two algorithms.

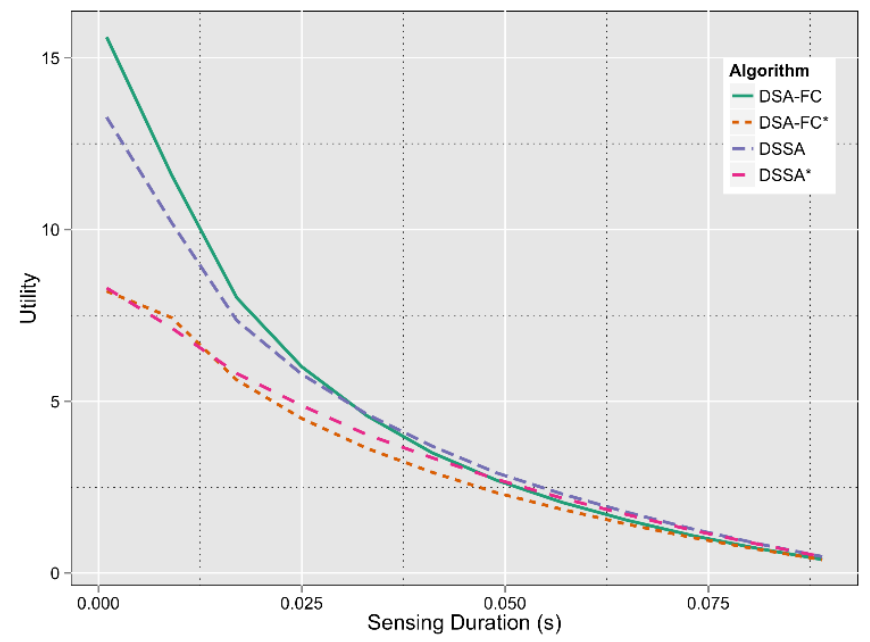

Fig. 13. Average SU utility vs. sensing duration.

\section{CONCLUSION}

In this paper, approaches to open spectrum sharing were outlined and detailed. A spectrum model for multi-channel dynamic spectrum access was presented and an in-depth analytical account of spectrum sensing was given. The details of a collaborative spectrum sharing game were then presented from an analytical perspective, and an adapted algorithm for fast convergence in a hedonic coalition game was given. Finally, the performance of the new algorithm was compared to the original algorithm and it was shown that the new algorithm outperforms the original one in terms of convergence time. This has strong implications in industrial settings because faster convergence time means shorter network setup time and greater scalability as the size of the network grows. Possible future work includes evaluating the algorithm with a dynamic channel occupancy model. 


\section{REFERENCES}

[1] T. Chiwewe and G. Hancke, "A look at spectrum management policies for radio spectrum," EngineerIT, no. March, pp. 47-49, 2015.

[2] L. Berlemann and S. Mangold. "Radio spectrum today - regulation and spectrum usage," in Cognitive Radio and Dynamic Spectrum Access Anonymous 2009, . DOI: 10.1002/9780470754429.ch2.

[3] T. M. Chiwewe, C. F. Mbuya and G. P. Hancke, "Using Cognitive Radio for Interference-Resistant Industrial Wireless Sensor Networks: An Overview," IEEE Trans. Ind. Informat., vol. 11, no. 6, pp. 1466-1481, 2015.

[4] A. Willig, "Recent and emerging topics in wireless industrial communications: A selection," IEEE Trans. Ind. Informat., vol. 4, no. 2, pp. 102-122, 2008.

[5] B. Silva, R. M. Fisher, A. Kumar and G. P. Hancke, "Experimental link quality characterization of wireless sensor networks for underground monitoring," IEEE Transactions on Industrial Informatics, vol. 11, no. 5, pp. 1099-1110, 2015.

[6] B. Cheng, L. Cui, W. Jia, W. Zhao and G. P. Hancke, "Multiple Regions of Interest Coverage in Camera Sensor Networks for Tele-Intensive Care Units," .

[7] K. S. E. Phala, A. Kumar and G. P. Hancke, "Air Quality Monitoring System Based on ISO/IEC/IEEE 21451 Standards," IEEE Sensors Journal, vol. 16, no. 12, pp. 5037-5045, 2016.

[8] B. Wang, Y. Wu and K. J. R. Liu, "Game theory for cognitive radio networks: An overview," Computer Networks, vol. 54, no. 14, pp. 2537 2561, 2010.

[9] Z. Shu, Y. Qian, Y. L. Yang and H. Sharif, "A game theoretic approach for energy-efficient communications in multi-hop cognitive radio networks," Wireless Commun. Mobile Comput., vol. 16, no. 14, pp. 2131-2143, 2016

[10] Y. Xiao, D. Niyato, Z. Han and K. -. Chen, "Secondary users entering the pool: A joint optimization framework for spectrum pooling," IEEE J Sel Areas Commun, vol. 32, no. 3, pp. 572-588, 2014

[11] X. Feng, H. Wang and X. Wang, "A game approach for cooperative spectrum sharing in cognitive radio networks," Wireless Commun. Mobile Comput., vol. 15, no. 3, pp. 538-551, 2015.

[12] J. Rajasekharan and V. Koivunen, "Cooperative game-theoretic approach to spectrum sharing in cognitive radios," Signal Process, vol. 106, pp. 15-29, 2015

[13] L. Yang, H. Kim, J. Zhang, M. Chiang and C. W. Tan, "Pricing-based decentralized spectrum access control in cognitive radio networks," IEEE ACM Trans Networking, vol. 21, no. 2, pp. 522-535, 2013.

[14] Y. Xu, A. Anpalagan, Q. Wu, L. Shen, Z. Gao and J. Wang, "Decisiontheoretic distributed channel selection for opportunistic spectrum access: Strategies, challenges and solutions," IEEE Commun. Surv. Tutor., vol. 15, no. 4, pp. 1689-1713, 2013.

[15] Y. Liu and L. Dong, "Spectrum sharing in MIMO cognitive radio networks based on cooperative game theory," IEEE Trans. Wireless Commun., vol. 13, no. 9, pp. 4807-4820, 2014.

[16] S. Gong, P. Wang and L. Duan, "Distributed power control with robust protection for PUs in cognitive radio networks," IEEE Trans. Wireless Commun., vol. 14, no. 6, pp. 3247-3258, 2015.

[17] G. Yang, B. Li, X. Tan and X. Wang, "Adaptive power control algorithm in cognitive radio based on game theory," IET Commun., vol. 9, no. 15, pp. 1807-1811, 2015.

[18] C. Chen, M. Song, C. Xin and J. Backens, "A game-theoretical antijamming scheme for cognitive radio networks," IEEE Network, vol. 27, no. 3, pp. 22-27, 2013.

[19] K. Dabcevic, A. Betancourt, L. Marcenaro and C. S. Regazzoni, "A fictitious play-based game-theoretical approach to alleviating jamming attacks for cognitive radios," in ICASSP, IEEE International Conference on Acoustics, Speech and Signal Processing - Proceedings, 2014, pp. 8158-8162.
[20] K. Dabcevic, A. Betancourt, L. Marcenaro and C. S. Regazzoni, "Intelligent cognitive radio jamming - A game-theoretical approach," Eurasip. J. Adv. Sign. Process., vol. 2014, no. 1, pp. 1-18, 2014.

[21] Y. Xiao, Z. Han, K. -. Chen and L. A. Dasilva, "Bayesian hierarchical mechanism design for cognitive radio networks," IEEE J Sel Areas Commun, vol. 33, no. 5, pp. 986-1001, 2015.

[22] O. Habachi, R. El-Azouzi and Y. Hayel, "A stackelberg model for opportunistic sensing in cognitive radio networks," IEEE Trans. Wireless Commun., vol. 12, no. 5, pp. 2148-2159, 2013.

[23] C. Jiang, Y. Chen, Y. Gao and K. J. R. Liu, "Joint spectrum sensing and access evolutionary game in cognitive radio networks," IEEE Trans. Wireless Commun., vol. 12, no. 5, pp. 2470-2483, 2013.

[24] C. Jiang, Y. Chen and K. J. R. Liu, "Multi-channel sensing and access game: Bayesian social learning with negative network externality," IEEE Trans. Wireless Commun., vol. 13, no. 4, pp. 2176-2188, 2014.

[25] X. Hao, M. H. Cheung, V. W. S. Wong and V. C. M. Leung, "Hedonic coalition formation game for cooperative spectrum sensing and channel access in cognitive radio networks," IEEE Trans. Wireless Commun., vol. 11, no. 11, pp. 3968-3979, 2012.

[26] Q. Zhao and B. M. Sadler, "A survey of dynamic spectrum access," IEEE Signal Process. Mag., vol. 24, no. 3, pp. 79-89, 2007.

[27] Y. Liang, Y. Zeng, E. C. Peh and A. T. Hoang, "Sensing-throughput tradeoff for cognitive radio networks," IEEE Transactions on Wireless Communications, vol. 7, no. 4, pp. 1326-1337, 2008.

[28] I. F. Akyildiz, B. F. Lo and R. Balakrishnan, "Cooperative spectrum sensing in cognitive radio networks: A survey," Physical Communication, vol. 4, no. 1, pp. 40-62, 2011.

[29] N. Zhang, H. Liang, N. Cheng, Y. Tang, J. W. Mark and X. S. Shen, "Dynamic spectrum access in multi-channel cognitive radio networks," IEEE J Sel Areas Commun, vol. 32, no. 11, pp. 2053-2064, 2014.

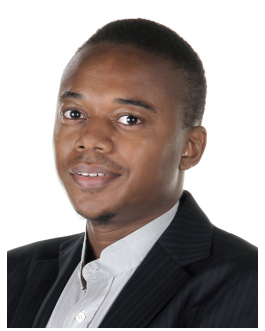

Tapiwa M. Chiwewe (S'09-M'13) received the B.Eng., M.Eng. and $\mathrm{PhD}$ degrees in computer engineering from the University of Pretoria (South Africa) in 2006, 2010, and 2016 respectively. He was a Software Developer at Fifth Dimension Technologies from 2008 to 2011, and a Senior Research Engineer at the Council for Scientific and Industrial Research (South Africa) from 2011 to 2015 . He is currently a Research Scientist and Manager at IBM Research in Johannesburg, South Africa. His research interests include cognitive radio networks and machine learning.

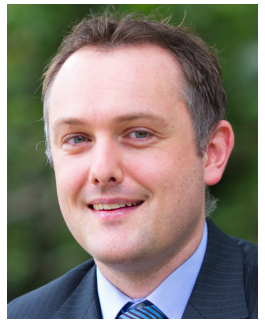

Gerhard. P. Hancke (S'99-M'07-SM'11) is an Assistant Professor with the City University of Hong Kong (Hong Kong $\mathrm{SAR}$ ). His research interests are system security, embedded platforms and distributed sensing applications. $\mathrm{He}$ obtained B.Eng. and M.Eng. degrees from the University of Pretoria (South Africa) in 2002 and 2003, and a PhD in Computer Science with the Security Group at the University of Cambridge's Computer Laboratory in 2008. 